\title{
Emociones, racionalidad y responsabilidad ${ }^{1}$
}

\author{
CARLOS J. MOYA
}

Universidad de Valencia

EN El ÁmBito de la filosofía de la mente y de la acción de orientación analítica, las emociones han recibido, en comparación con otros tipos de estados y procesos mentales, escasa atención. Una razón de este estado de cosas es el predominio del paradigma cognitivo y del funcionalismo computacional, en cuyo marco los procesos mentales son concebidos como operaciones formales con representaciones, con independencia de la base física que lleva a cabo tales operaciones. Las emociones encuentran difícil acomodo en esta concepción de la mente, debido a la importancia que cobran en ellas, por un lado, estructuras y procesos fisiológicos específicos, $\mathrm{y}$, por otro, aspectos de carácter fenomenológico, los llamados qualia. Así, o bien las emociones han sido consideradas como excrecencias atípicas más o menos intratables y relegadas a zonas marginales de la vida mental, o bien han tendido a ser asimiladas a estados representacionales, concibiendo los aspectos fisiológicos y fenomenológicos como acompañantes inesenciales de las mismas. Ninguna de estas dos opciones resulta satisfactoria. La segunda da lugar a una

1 El presente trabajo procede del tex to de la ponencia que presenté en el congreso "Analytic Philosophy at the Turn of the Millennium", celcbrado en Santiago de Compostela en diciembre de 1999. Constituye una revisión importante de una ponencia anterior, titulada "Emociones y libertad", que expuse en el congreso "Emociones". celebrado en Málaga en abril de 1999. Agradezco al profesor Martínez. Freire su invitación a colaborar en el presente número de la revista Contrastes. El trabajo se inserta en el marco del proyecto PB96-1091-C03-02, otorgado por la DGES. 
imagen extremadamente abstracta de la mente humana, en la que difícilmente podríamos reconocernos. En cuanto a la primera, entra en claro conflicto con la omnipresencia e importancia de las emociones en nuestra vida mental, sobre todo si incluimos en ellas los estados de ánimo, lo que cabría llamar el tono emocional que permea en cada momento nuestra actividad y nuestra relación con las cosas y las personas. En una vida emocional satisfactoria se encuentra gran parte de la clave de la felicidad humana, y los seres humanos tenemos derecho a esperar de la filosofía que contribuya a ella a través de un conocimiento más profundo de nuestra naturaleza. Una concepción de los seres humanos en la que las emociones ocupen el lugar que corresponde a su importancia en nuestras vidas sería un paso en esta dirección. La reflexión filosófica, sin embargo, no debería permitirse a sí misma, en su búsqueda de la verdad, la arrogancia de ignorar o menospreciar el esfuerzo intelectual llevado a cabo en otras disciplinas, como la psicología y la neuropsicología. Hay problemas comunes a la fílosofía y a estas ciencias, y muchos de los avances en estos campos son claramente pertinentes para la reflexión y la argumentación filosófica. Por otra parte, un estudio en profundidad de la vida emocional, en el espíritu indicado, puede contribuir, en mi opinión, a transformar el planteamiento de algunas cuestiones fïlosóficas centrales, como la relación entre la mente, el cerebro y el cuerpo, la identidad personal, la memoria, la posibilidad del conocimiento o la libertad y la responsabilidad moral.

En esta ponencia quisiera ocuparme de esta última cuestión, abordando algunos problemas que plantean las emociones a la posibilidad de la responsabilidad moral.

Es un hecho que, con frecuencia, juzgamos moralmente a las personas en virtud de sus emociones y, con ello, las consideramos de algún modo responsables de ellas ${ }^{2}$. Por otra parte, es también un hecho que en ocasiones nos sentimos culpables por experimentar ciertas emociones, lo que significa que en algún grado nos consideramos (correctamente o no) responsables de tenerlas. Pero, en general, al atribuir responsabilidad a un sujeto presuponemos que posee algún grado de control voluntario sobre el objeto de esa responsabilidad, presuponemos que hay algún sentido en que dependía de él, es decir, algún sentido en que el agente poseía libertad en relación con ese objeto. Así, pues, en la medida en que hacemos a un sujeto responsable de sus emociones, presuponemos que

2 Cl., p. ej, R. De Sousa, 'The Rationality of Emotion', en A. Rorly (ed.). Explaining Emotions, University of California Press, Berkeley, 1980, p. 141. 
posee algún grado de control voluntario, de libertad en relación con ellas. Sin embargo, este supuesto no es obviamente verdadero, sino que requiere justificación. Y a menos que la tenga, las práctica; de atribución de responsabilidad que descansan en él podrían asimismu estar injustificadas, aun cuando seguramente esta falta de justificación no haría que renunciásemos a ellas.

Ahora bien, el mero control voluntario no es suficiente para la responsabilidad. Un perro, por ejemplo, controla voluntariamente sus movimientos, pero no lo hacemos a él responsable de sus actos. No lo consideramos un ser libre. La libertad y el control voluntario que dan cabida a la responsabilidad requieren la posesión de una voluntad racional, de una capacidad para la deliberación práctica y la toma de decisiones entre cursos de acción alternativos sobre la base de razones. De ahí que las acciones que son el resultado de un proceso de razonamiento práctico de ese tipo sean paradigmas de aquello por lo que consideramos a un sujeto responsable.

No parece que las emociones se incluyan en la categoría de las acciones. Su denominación más tradicional, «pasiones», alude precisamente a su carácter pasivo ${ }^{3}$. Las emociones parecen ser ciertamente estados o procesos que tienen lugar en nosotros, no cosas que hacemos. Sin embargo, aun cuando parezca extraño, hay filósofos que se oponen a esta concepción pasiva de las emociones, adoptando de este modo una línea radical en lo que respecta a la relación entre responsabilidad y emociones. Un representante de esta línea es Robert Solomon4. Según Solomon, «las emociones no son aconteceres, no son cosas que nos suceden. Quisiera sugerir que las emociones son racionales y propositivas, más que irracionales y disruptivas; son muy semejantes a acciones, y elegimos una emoción en gran medida como elegimos un curso de acción»5. Estas declaraciones altisonantes se ven fuertemente matizadas, sin embargo, cuando Solomon aclara que las emociones guardan una interesante similitud con las creencias y que son, en realidad, juicios normativos y a menudo morales sobre situaciones en que nos hallamos ${ }^{6}$. Desde este punto de vista, las emociones poseen, como juicios que son, contenido intencional de carácter proposicional. Los juicios en que consisten las

3 Aunque la denominación «emociones» incluye la idea de aclividad. de un 'movimiento desde' (e-movere).

4 El iniciador de esta línea es Jean Paul Sartre.

5 Solomon, R. C., 'Emotions and Choice', en A. O. Rorty (cd.), Explaining' Emotions, pp. 252-81, esp. pp. 251-2.

$6 \mathrm{Cl}$. ibid., p. 257. 
emociones expresan nuestras creencias. Solomon acepta que no podemos simplemente elegir estar enfadados o no, sino que, para conseguir estarlo o no, hemos de llevar a cabo otras actividades. Pero señala que esto mismo sucede con nuestros juicios en general y, en realidad, con la mayoría de nuestras acciones: «No puedo simplemente asesinar a un dictador. He de hacer alguna otra cosa (presionar el gatillo de un rifle...) ${ }^{7}$. Solomon vuelve a insistir, pues, en el carácter de acciones propio de las emociones, pero es obvio que el paralelismo que traza entre emociones y acciones en este punto es inadecuado. Mientras que es plausible sostener que presionar el gatillo de un rifle y matar a un dictador no son dos acciones particulares que llevo a cabo, sino dos descripciones de una única acción en términos de sus consecuencias o efectos 8 , contar hasta diez, digamos, no es otra descripción de dejar de estar enfadado. Solomon insiste en que los juicios son algo que hacemos, pero esta consideración no resuelve nada, porque también podríamos decir que digerir alimentos es algo que hacemos, sin que ello convierta la digestión en una acción. El propio Solomon reconoce este punto en un apéndice a su trabajo original, donde suaviza también otros aspectos de su posición inicial. Una objeción obvia (y en mi opinión correcta) a su posición es que se puede formular el juicio normativo y no tener la emoción: se puede, por ejemplo, juzgar que algo es peligroso y no sentir miedo. En respuesta a ello, Solomon no identifica ya una emoción simplemente con un juicio, sino con un sistema de juicios, de modo que, según él, no es posible llevar a cabo este sistema de juicios y no sentir la emoción. Pero esta afirmación necesitaría una prueba que no se nos ofrece. Solomon, finalmente, acaba poniendo en suspenso su tesis inicial según la cual las emociones son voluntarias y objeto de elección. Sin embargo, sigue manteniendo que tienen, como los juicios, explicaciones por razones, en forma de otros juicios. y que somos responsables de ellas como lo somos de nuestros juicios.

Puesto que el mismo Solomon se retracta de su asimilación de las emociones a acciones, no nos detendremos mucho en rechazar esta tesis, que apenas encuentra partidarios en la actualidad. Como señala Elster, esta tesis no es sólo contraria a nuestras intuiciones, sino sencillamente falsa ${ }^{9}$. Puede

7 Ibid., p. 261.

8 Por ejemplo, desde una concepción davidsoniana de la individuación de las. acciones.

9) I. Elster, Alchemies of the Mind. Rationality and the Emotions, Cambridece Universily Press, Cambridge, 1999, p. 311. Cl. th. LeDoux: «... Las cmociones son cosas (jue nos suceden, más que cosas que queremos que ocurran... Tenemos escaso control directo sobre nuestras reacciones emocionales》. (The Emotional Brain. Simon \& Schuster, New York, 1998, p. 19). 
que las emociones incluyan un componente de actividad, en tanto que pueden concebirse, en parte, como respuestas o reacciones ante situaciones u objetos, pero no constituyen casos de conducta intencional. Puede que sean o incluyan respuestas o reacciones, pero no son acciones.

Ahora bien, la defensa de la responsabilidad por nuestras emociones no exige realmente, desde el punto de vista de nuestras propias prácticas de atribución de responsabilidad, asimilarlas implausiblemente a acciones. De hecho, atribuimos responsabilidad a las personas también por actos y omisiones involuntarias, así como por meros sucesos. Así, por ejemplo, podemos atribuir justamente responsabilidad a un amigo por olvidar acudir a una cita, aun cuando el olvido no fue voluntario. Igualmente, cabe hacer a un guardabarreras responsable de un accidente si, por ejemplo, estaba dormido cuando debía hacer bajar las barreras. aun cuando el accidente fue un mero suceso, no una acción. Estos ejemplos no muestran, sin embargo, que la responsabilidad sea independiente de la libertad y del control voluntario racional de las propias acciones. En casos como éstos suponemos que estaba en poder del agente, al menos en algún momento anterior, haber hecho algo sobre lo que tenía control voluntario directo y que, previsiblemente, habría evitado aquello de lo que le hacemos responsable. El guardabarreras, por ejemplo, podría haber puesto los medios necesarios para evitar quedarse dormido. Y algo semejante cabe decir del otro ejemplo. Si este supuesto no sc da, consideramos injustificada la atribución de responsabilidad. Este sería el caso si, por ejemplo, el guardabarreras se quedó dormido porque alguien puso un potente somnífero en su café sin que él lo advirtiera. El control voluntario, pues, es condición de la responsabilidad, pero no es necesario que el agente tenga un control voluntario directo de aquello por lo que se le considera responsable. El control puede ser indirecto, es decir, derivado de actos que controla directamente. Y este control indirecto admite cierto grado de falibilidad.

Si las emociones no son acciones, el control que podemos tener sobre ellas ha de ser, en el mejor de los casos, indirecto. En realidad, la pretensión de tener control voluntario directo de determinados estados es en sí misma irracional. Pensemos por ejemplo en nuestras creencias. La actitud adecuada ante nuestras creencias, si pretendemos que scan verdaderas, es permitir que vengan determinadas por el modo en que las cosas son realmente, no por nuestra voluntad. $\mathrm{O}$, dicho de otro modo, la voluntad racional con respecto a nuestras creencias es que respondan a la realidad, no a nuestros deseos. El llamado pensamiento desiderativo o 'wishful thinking' es un fenómeno irracional. Sin embargo, se puede hacer justificadamente responsable a un sujeto de sus creencias en la 
medida en que éstas dependan de algo que el sujeto hace o pudo hacer voluntariamente, por ejemplo investigar más, recoger mayor información, razonar de modo más cuidadoso, etc., precisamente con vistas a asegurar al máximo su verdad.

Podemos preguntarnos ahora si la responsabilidad que podemos tener con respecto a nuestras emociones es del tipo de la que podemos tener con respecto a nuestras creencias. El control que podemos ejercer sobre nuestras creencias a través de nuestra actividad de investigación, razonamiento, etc., depęnde de la existencia de relaciones deductivas o de 'probabilificación' entre contenidos proposicionales, por ejemplo entre los contenidos proposicionales de la percepción cognitiva y de las creencias que formamos sobre la base de aquélla ${ }^{10}$. Las relaciones causales que conducen a la formación de creencias han de estar basadas en tales relaciones inferenciales entre contenidos para que nuestras creencias estén racionalmente justificadas. En realidad, como he arguiido en otros trabajos, el concepto de justificación es parcialmente causal. " La concepción cognitiva de las emociones, si fuera correcta, permitiría un control similar sobre ellas que justificaría la atribución de responsabilidad. Analicemos ahora esta propuesta.

La concepción cognitiva de las emociones, que se remonta a Aristóteles, puede adoptar versiones más o menos fuertes 12 . En sus versiones más fuertes o reductivas, las emociones son identificadas con creencias o juicios cuyo contenido es parcialmente normativo o evaluativo. R. S. Peters defiende esta tesis cuando afirma: «Sentir miedo es, por ejemplo, ver una situación como peligrosa»13. La posición suavizada de Solomon, tras rechazar la propuesta según la cual las emociones serían acciones voluntarias y objeto de elección, sería de este tipo. También cabría encuadrar aquí las concepciones del estoicismo y de Spinoza. En sus versiones más débiles, no reductivas, las emociones requieren creencias como causas o constituyentes parciales. En palabras de Hansberg: «Una teoría cognoscitiva deberá sostener... que sin una creencia característica

10 Cl. J. McDowell, Mind and World, Harvard University Press, Cambridge MA. 1994, Lecture I.

11 Cf. mi 'Justificación, causalidad y acción intencional', Theoria 13 (1998), pp. 349-65 y 'Reason and Causation in Davidson's Theory of Action Explanation', Crítica 30 (1998), pp. 29-43.

12 Sobre esta distinción, véase C. DeLancey, 'Real Emotions', Philosophical Psychology 11 (1998), pp. 467-87, esp. p. 468.

13 R. S. Peters, 'The Education of Emotions', cit. en W. Lyons, Emotion. Cambridge University Press, Cambridge, 1980, p. 51. 
no hay una determinada emoción»14. La concepción aristotélica pertenece a esta clase. También Olbeth Hansberg sostiene una posición semejante en su reciente libro sobre las emociones. Las teorías cognitivas consideran el contenido intencional como el criterio esencial de individuación de las emociones; los aspectos fenomenológicos y fisiológicos pueden o no estar presentes, pero no son realmente esenciales.

La concepción cognitiva permite la posibilidad de un control racional sobre las emociones semejante al que podemos tener sobre las creencias. Al dotar las emociones de contenido proposicional, o hacerlas depender de estados que lo poseen, las emociones pueden entrar en el mismo tipo de relaciones inferenciales y causales que permiten la justificación de nuestras creencias y pueden ser indirectamente controladas por nuestra actividad cognoscitiva voluntaria. En el marco del funcionalismo computacional, las emociones, como estados representacionales, pueden ser integradas en el procesamiento de información que constituye, para esta perspectiva, la actividad mental. En el marco del tratamiento de las perturbaciones emocionales, diversas formas de terapia psicológica, en particular la terapia racional-emotiva ${ }^{15}$, presuponen la validez de la concepción cognitiva de las emociones. Igualmente, la actual proliferación de cursos sobre inteligencia emocional, a partir del best-seller de Daniel Goleman ${ }^{16}$, descansa fundamentalmente en dicha concepción.

Esta concepción de las emociones es optimista por lo que respecta a la libertad y la responsabilidad de que gozamos en relación con nuestros estados y procesos emocionales. Somos responsables de nuestras emociones de un modo semejante a como lo somos respecto de nuestras creencias y, como en este último caso, podemos ejercer una actividad voluntaria dirigida a poseer emociones racionales, adecuadas a nuestro mejor juicio sobre la situación en que nos hallamos.

Sin embargo, hay, en mi opinión, importantes razones para el escepticismo con respecto a la concepción cognitiva de las emociones y para moderar el optimismo asociado a ella sobre la posibilidad del control racional de nuestras emociones. La concepción tradicional que considera las emociones como fuerzas ajenas a la razón y la libertad y en conflicto potencial con ellas puede contener importantes elementos de verdad. Es difícil dar sentido a determinados procesos irracionales, como la

14 Hansberg, O., La diversidad de las emociones, F. C. E., México, 1996, p. 115. $15 \mathrm{Cl}$. A. Ellis y R. Grieger (eds.), Manual de terapia racional-emotiva (4a. edición), Desclée de Brouwer, Bilbao, 1985.

16 D. Goleman, Inteligencia emocional, Kairós, Barcelona, 1996. 
akrasia, el pensamiento desiderativo (y el pensamiento contradesiderativo) ${ }^{17}$, el autoengaño o la cristalización ${ }^{18}$, entre otros, sin apelar al papel perturbador de las emociones en los procesos cognitivos. Veamos algunas consideraciones contrarias al cognitivismo.

En primer lugar, desde el punto de vista evolutivo, los sistemas de respuesta emocional son más primitivos que los sistemas cognitivos superiores y mantienen sus propias vías de relación con los sistemas sensoriales. Como señala LeDoux, compartimos los sistemas neurofisiológicos vinculados a determinadas emociones básicas, como el miedo o la atracción sexual, con especies situadas muy por debajo de nosotros en la escala evolutiva ${ }^{19}$. De ahí que los estudios realizados sobre miembros de estas especies tengan utilidad para la comprensión de las emociones humanas. La respuesta de miedo involucra de modo esencial la amígdala, por ejemplo, en especies muy diversas. Pero la amígdala tiene sus propias vías de conexión con las terminaciones sensoriales. Así, los estímulos sensoriales son transmitidos al tálamo sensorial, y a partir de él surgen vías que transmiten la señal directamente a la amígdala, mientras que otras vías se dirigen al córtex sensorial y a través de éste a la amígdala. Hay, pues, dos vías de transmisión, la una directa y la otra indirecta, desde el tálamo sensorial a la amígdala. Como señala LeDoux, la vía directa o inferior «sólo puede proporcionar a la amígdala una representación burda del estímulo. Es, pues, una vía de procesamiento rápida y borrosa»20. Por el contrario, la vía indirecta o superior es más lenta, pero más precisa. Ahora bien, es difícil pensar que la señal que llega directamente a la amígdala sea interpretable (descodificable) en forma de un contenido proposicional, a menos que deseemos atribuir también esta clase de contenido a las ratas. La existencia de una vía rápida que desencadena las respuestas emocionales antes de que la señal sensorial haya sido procesada por el córtex tiene sentido desde el punto de vista evolutivo. Como LeDoux lo expresa: «El tiempo ganado por la amígdala al actuar sobre la base de la información talámica, en lugar de esperar a la entrada procedente del córtex, puede significar la diferencia

17 Sobre el pensamiento contradesiderativo, un fenómeno ciertamente intrigantc, cf. Elster, Alchemies..., secciones I.6, II.3, III.3.

18 Este lenómeno, relacionado con la emoción del amor, «es un modo de formación irracional de creencias, que induce una creencia exagerada en la belleza y méri10' de la otra persona... la emoción trabaja retrospectivamente, para inventar su propia justilicación» (Elster, Alchemies..., pp. 129-30)

$19 \mathrm{Cf}$. LeDoux, The Emotional Brain..., p. 17.

$20 \mathrm{lbid}$, p. 164. 
entre la vida y la muerte. Es mejor haber tratado un palo como una serpiente que no haber respondido a una posible serpiente»2!. Pensemos, sin embargo, que estas afirmaciones contradicen el principio básico de la concepción cognitiva de las emociones, según el cual no hay una emoción sin una creencia característica, puesto que la reacción de miedo comienza antes de que se haya formado una creencia proposicional, conceptualmente estructurada, en el nivel cortical. Es este un punto en el que la neurofisiología se enfrenta a intuiciones que pueden parecer obvias, como que el miedo requiere la creencia en la existencia de un peligro. Estas intuiciones muestran un marcado sesgo antropocéntrico.

Por otra parte, LeDoux presenta el caso de pacientes cuyos hemisferios cerebrales no se comunican entre sí, debido a que las conexiones han sido seccionadas con el fin de controlar una epilepsia severa. Las pruebas con uno de estos pacientes mostraban que, en determinadas condiciones, el sujeto (o uno de sus hemisferios cerebrales) era capaz de juzgar la significación emocional de un estímulo (si el estímulo era, digamos, bueno o malo) sin poder juzgar de qué estímulo se trataba, es decir, sin ser capaz de conceptuar cognoscitivamente el estímulo. El sujeto sabía que el estímulo era algo malo (o bueno), pero no podía decir qué era. El procesamiento emocional del estímulo tenía lugar, presumiblemente, de modo no consciente y con independencia de su procesitmiento cognitivo. De nuevo, lo que esto sugiere es que la creencia no es una condición indispensable de la emoción. Por otra parte, los sistemas emocionales parecían operar en paralelo con los sistemas cognitivos y utilizar un código distinto del empleado por éstos. Como LeDoux señala, «los sistemas no verbales e inconscientes... constituyen el corazón y el alma de la máquina emocional»22. En este punto, es difícil no pensar en la distinción de Freud entre el proceso primario, el lenguaje no verbal del insconciente, y el proceso secundario, propio del yo consciente. Esta distinción podría encontrar un fuerte apoyo en los hallazgos de la neuropsicología. Desde el punto de vista evolutivo, es plausible pensar que el funcionamiento de los sistemas emocionales primarios, relacionados con la supervivencia y la reproducción, no podía esperar al desarrollo de los sistemas cognitivos superiores y del pensamiento verbal y conceptual. En los seres humanos, el desarrollo de los sistemas cognitivos vinculados al córtex se habría producido sin modificaciones esenciales en los sistemas de respuesta emocional. El conflicto, muchas veces do-

21 lbid., p. 166.

22 lbid., p. 72. 
loroso, entre la pasión y la razón encuentra así una base material en las contingencias de la evolución biológica ${ }^{23}$.

Las emociones, a diferencia de las creencias, involucran partes del cuerpo distintas del cerebro y del sistema nervioso, en particular el sistema hormonal. La secreción de determinadas glándulas influye retroactivamente en el sistema nervioso 24 . Pero es difícil pensar que estas relaciones entre el sistema hormonal y el sistema nervioso se hallen mediadas por representaciones. Como Rey señala: «Me parece que, hasta donde podemos saber, no tenemos razón alguna para creer que las acciones e interacciones del sistema hormonal con el sistema cognitivo involucren en modo alguno relaciones racionales entre representaciones. Las relaciones que se dan parecen ser meramente causales, semejantes a este respecto a las que están implicadas en la ósmosis... Ciertas propiedades lisa y llanamente físicas de nuestra corporeidad pueden ser, muy probablemente, esenciales a nuestra mente» 25 . Estas observaciones, si son correctas, constituyen un serio obstáculo para la versión específica del cognitivismo que es el funcionalismo computacional: las emociones son más dependientes de la fisiología efectiva de las especies de lo que el funcionalismo computacional puede permitir. Pero la dificultad afecta también a una concepción cognitiva más general de las emociones, y con ella a la posibilidad de un control racional sobre ellas. La actividad cognitiva voluntaria podría tener un efecto sumamente limitado sobre los estados emocionales si éstos involucran relaciones meramente causales, no mediadas por representaciones, entre, por ejemplo, el sistema nervioso y el sistema hormonal del organismo. Una diferencia importante a este respecto es que, mientras que nos es posible esperar a formar una determinada creencia (suspender el juicio) hasta que tengamos, por ejemplo, mayor información, no podemos, en este sentido, esperar a formar una emoción. Las reacciones emocionales se

23 En este mismo sentido cabe mencionar la distinción de LeDoux entre la memoria emocional, de carácter implícito pero altamente persistente, relacionada esencialmente con la amígdala, y la memoria de emociones, de carácter explícito y vinculada al lóbulo temporal y el hipocampo. Esta distinción puede dar cuenta de determinadas patologías relacionadas con traumas emocionales. Pero no vamos a entrar más a fondo en ello.

24 Este aspecto de las emociones, el llamado 'feedback' corporal, fue subrayado fuertemente por William James.

25 G. Rey, 'Functionalism and the Emotions', en Rorty (ed.), Explaining Emotions. pp. 163-95, esp. p. 191. Este punto sugiere el modo en que las emociones podrían transformar el problema de la relación mente/cuerpo. Pensemos también en sus consccuencias para el experimento mental putnamiano de los cerebros en cubetas. 
desencadenan de modo más o menos automático, sin esperar a los resultados de nuestras investigaciones o de nuestro razonamiento.

Además, y esta es una observación independiente de la neurofisiología, pero congruente con ella, aunque algunas emociones (especialmente las llamadas secundarias) puedan tener contenido proposicional, muchas de ellas, y en particular las de carácter primario, como el miedo, la atracción sexual o el asco (la repugnancia), no lo tienen, sino que su contenido es un objeto (o la representación del mismo). Podemos tener miedo o asco de un objeto, y sentirnos atraídos sexualmente por una persona. Como escribe Elster: «Los objetos de las creencias y los deseos son siempre proposiciones... Los objetos de las emociones pueden ser proposiciones, pero no es necesario que lo sean... La emoción de la ira puede vincularse a un objeto personal o a uno proposicional. Pero en el caso del odio y el amor, la emoción sólo puede formularse con un objeto personal»26. Estas consideraciones no son nuevas, pero sus consecuencias para la concepción cognitiva y la responsabilidad por las emociones no han sido en general tenidas en cuenta. Pensemos, a este respecto, que las relaciones lógicas o de 'probabilificación' se dan únicamente entre proposiciones; no hay tales relaciones entre representaciones de objetos y contenidos proposicionales. Pero esto es precisamente lo que se necesitaría para que fuésemos responsables de nuestras emociones en el sentido en el que podemos serlo de nuestras creencias. La entrada que desencadena una emoción no tiene por qué ser una creencia proposicional; puede ser la representación sensorial de un objeto. Una vez más, la tesis mínima del cognitivismo, según la cual no hay emoción sin creencia, no parece ser cierta.

Finalmente, determinados estados de ánimo, en la medida en que puedan considerarse estados emocionales, y determinadas emociones estéticas constituyen nuevas dificultades para la concepción cognitiva. Muchos estados de ánimo (moods), como se reconoce generalmente, carecen de contenido intencional, aunque puedan tener causas. Así, aunque en ocasiones sus causas puedan ser cognitivas, la relación no puede incluir un elemento inferencial. En cuanto a las emociones estéticas, pensemos en el sentimiento de alegría o tristeza que puede generar una pieza musical. La emoción no se halla aquí generada por una evaluación cognitiva27. Aun

26 Elster, Alchemies..., p. 271.

27 Las emociones generadas por la música constituyen un contraejemplo a las llamadas teorías de la valoración (appraisal), representadas, entre otros, por Nico Frijda o Richard S. Lazarus. Cl. Elster, Alchemies..., pp. 245-6. Cl. N. H. Frijda (ed.), Appraisal and Beyond. The lssue of Cognitive Determinants of Emotion, numero especial de Cognition and Emotion, Lawrence Erlbaum Associates, Hillsdale, 1993. 
suponiendo que pudiera arguiirse que, incluso en este caso, la emoción estaría generada por alguna representación (si entendemos como tal la percepción auditiva), lo que se necesitaría para fundamentar la responsabilidad por las emociones serían respresentaciones capaces de entrar en las relaciones inferenciales que permiten el control racional de las propias creencias. Y no parece ser éste el caso, no solamente por lo que respecta a las emociones generadas por la música, sino a muchas otras, según hemos indicado 28 .

En esta situación, es tentador pensar en el ideal estoico de la apátheia, de una vida libre de emociones, regida únicamente por propósitos libremente elegidos y creencias racionalmente justificadas. El problema con esta propuesta es que descansa precisamente en la misma concepción cognitiva cuyos problemas hemos puesto de manifiesto. Para el estoicismo, las emociones son fundamentalmente juicios parciales y sesgados sobre la vida y sobre nuestro lugar en el mundo, que generan expectativas injustificadas ante la realidad. La realidad, sin embargo, se halla fuera de nuestro control, de modo que esas expectativas se ven frecuentemente frustradas, lo que nos lleva a la intranquilidad y el constante desasosiego. Es el conocimiento de la futilidad de nuestros vínculos emocionales lo que puede conducirnos a la tranquilidad de ánimo. En el caso de Spinoza, es también el conocimiento de la verdadera naturaleza de las cosas, el amor intelectual de Dios, que nos permite apreciar que el mundo no está hecho para satisfacer nuestros deseos subjetivos, el que puede librarnos de la esclavitud de las pasiones y conducirnos a la libertad y a la autonomía. Sin embargo, las emociones podrían ser mucho menos sensibles a nuestra actividad cognitiva de lo que se supone en estas teorías, si los problemas de la concepción cognitiva que hemos señalado son reales. Por otra parte, el carácter presumiblemente innato de diversos sistemas emocionales y la capacidad motivadora de las emociones hace difícil pensar que la apátheia estoica sea una opción viable para los seres humanos.

El papel selectivo, parcial, de las emociones, el sesgo que introducen en nuestra relación con la realidad era ya, como vemos, un aspecto importante de la teoría estoica, y ha sido subrayado frecuentemente con posterioridad por distintos autores. Ben Ze'ev, por ejemplo, habla de «su naturaleza parcial y discriminatoria. No cualquier cosa o persona

28 Sería pertinente aquí aludir al contraste que Thomas Mann establece entre la música, un arte alemán por excelencia, y la literatura, un arte francés. racional y "democrático". 
tienen significación emocional para nosotros» 29 . En general, sin embargo, este rasgo de las emociones ha sido considerado fundamentalmente negativo para nuestra actividad racional, tanto teórica como práctica, en la medida en que nos impide alcanzar una apreciación objetiva de las cosas y las personas. Recientemente, sin embargo, diversos autores han sostenido una valoración contraria del sesgo emocional, del carácter selectivo de las emociones. Este es el caso de la «hipótesis del marcador somático» que Antonio Damasio propone en su importante libro El error de Descartes ${ }^{30}$. Según Damasio, la participación emocional en el proceso de deliberación práctica y toma de decisiones, lejos de constituir una interferencia perturbadora, es más bien una condición de posibilidad del mismo. Si nuestra deliberación práctica tuviese que desarrollarse según los criterios de la teoría de la decisión, no podríamos tomar decisiones adecuadas a las urgencias de la vida cotidiana, debido a la complejidad del cálculo requerido y al gran número de cursos de acción alternativos a evaluar. Este número, sin embargo, es drásticamente reducido, según Damasio, por la participación de las emociones en la selección de las alternativas que serán tenidas en cuenta. Esta participación ajusta además la evaluación de las alternativas a las peculiaridades afectivas de cada sujeto. Según la hipótesis del marcador somático, el córtex prefrontal elabora representaciones de las alternativas posibles, y estas representaciones son rápidamente evaluadas con la ayuda de las estructuras límbicas, que generan «sentimientos viscerales» (gut feelings). un remedo de las reacciones emocionales que despertarían en nosotros si fuesen reales. La evaluación emocional descarta con rapidez las alternativas que generan reacciones negativas y preselecciona como

29 A. Ben Ze'ev, 'Envy and Inequality', Journal of Philosophy' 89 (1992), pp. 551-81, esp. p. 558. Este carácter de las emociones es también un tema central en el libro de De Sousa sobre las emociones: «Durante un período de tiempo variable, pero siempre limitado, una emoción limita el campo de información que un organismo tendrá en cuenta, las inferencias que efectivamente extraerá entre una infinidad potencial y el conjunto de opciones pertinentes entre las que elegirá» (R. De Sousia, The Rationality. of Emotion, The MIT Press, Cambridge MA, 1987, p. 195). Y Nico Frijda escribe: "Las emociones involucran atribuciones, cambios de creencia, cambios en la atención y en la sensibilización hacia clases particulares de estímulos. No por castalidarl la emoción ha sido definida como un cambio en la activación "que, cuando se da. la mente es determinada con ello a pensar en una cosa más que en otra" (Spinorat...)» $(\mathrm{N}$. H. Frijda, 'The Place of Appraisal in Emotion', en N. Frijda (ed.), Appraisal cind Beyond..., pp. 357-87, esp. p. 371.

30 A. R. Damasio, El error de Descartes, Crílica, Barcelona, 1996. Edición original: Descartes' Error, G. P. Putnam, Nueva York, 1994. 
candidatas a la elección final aquellas que despiertan respuestas viscerales positivas. Los datos experimentales que maneja Damasio son, sin embargo, compatibles con otras explicaciones, y el propio Damasio propone la suya como una hipótesis 31.

En todo caso, si la hipótesis es correcta, y si las dudas que hemos expuesto sobre las posibilidades del control racional que podemos ejercer sobre nuestras emociones son fundadas, ello comprometería no sólo la justificación de las atribuciones de responsabilidad por nuestros estados emocionales, sino también por nuestras decisiones y acciones. El problema de la responsabilidad por nuestras emociones enlaza de este modo con los problemas tradicionales del libre albedrío y de la responsabilidad moral. En general, si no podemos ser realmente responsables de nuestras emociones, cuanto más destacado sea el papel que éstas desempeñan en la deliberación práctica, tanto más problemática será nuestra responsabilidad por nuestras decisiones y acciones. Por otra parte, si, por ejemplo, los sentimientos o sensaciones viscerales desempeñan el papel destacado que Damasio les concede, es de temer que las decisiones basadas en la prudencia o en la moralidad, en la medida en que contravengan nuestros deseos inmediatos, tenderán a ser descartadas en las fases iniciales de la deliberación.

Hay pocas dudas de que en una vida emocional en armonía con nuestras creencias y valores reside gran parte de nuestra felicidad y de la satisfacción con nosotros mismos. Pero el control que podemos tener sobre el logro de una armonía semejante depende en gran medida del control que podemos ejercer sobre nuestras emociones, de nuestra libertad con respecto a ellas. Si las consideraciones que preceden son correctas, este control es muy limitado. Aristóteles arguye que una actuación virtuosa, moderada, puede influir retroactivamente sobre las pasiones y producir equilibrio o armonía en las pasiones mismas 32 . Pero, aun cuando

31 Unos años antes, Ronald De Sousa había insistido asimismo en el papel positivo que la naturaleza selectiva de la emoción desempeña en el razonamiento práctico. Según De Sousa, sin la participación de las emociones, los procesos del razonamiento práctico y la toma de decisiones sucumbirían a lo que en inteligencia artificial se denomina «cl problema del marco» (frame problem). El problema consiste, en palabras de De Sousa, en «cómo hacer uso de los (conocimientos) que necesitamos entre el vasto conjunto (de los que tenemos) y en cómo no echar mano de aquellos que no necesilamos» (R. De Sousa, The Rationality of Emotion, p. 193). Las emociones, según De Sousa, serían el modo en que la naturaleza ha respondido al problema del marco en el caso de los seres humanos.

32 Cf. J. Sabini y M. Silver, 'Emotions, responsibility, and character'. en F. Schocman (cd.), Responsibility, Character; and the Emotions, Cambridge University Press, Cambridge, 1987, pp. 165-175, esp. p. 171. 
esta sugerencia pueda ser correcta en algunos casos, dista mucho de ofrecer una solución de carácter general a nuestro problema. Podemos ejercer un control sobre la conducta derivada de emociones que no aprobamos, cuando la intensidad de estas emociones es moderada, pero la emoción cuya expresión no permitimos no desaparece necesariamente con ello y puede generar un conflicto interno que eventualmente da lugar al desequilibrio psíquico. Esto no significa que la tarea de una educación emocional esté condenada al fracaso desde el principio. Pero significa que el conflicto potencial entre racionalidad y emociones, entre la razón y la pasión, por utilizar los términos tradicionales, puede ser en último término ineliminable. Una parte de esa educación consiste en aprender a vivir con ese conflicto, aceptando la precariedad de la condition humaine. Significa, en segundo lugar, que la educación emocional es un arte, y no una ciencia, un proceso sujeto a contingencias azarosas y en el que la suerte no deja de desempeñar un papel. La educación emocional no es comparable al aprendizaje epistémico si, como hemos argüido, las consideraciones racionales y las emociones no se relacionan entre sí del modo en que lo hacen las creencias. Las distintas formas de psicoterapia, el psicoanálisis, la terapia conductual, la terapia cognitiva, etc., funcionan unas veces, pero otras no lo hacen, y lo preocupante es que no sabemos bien a qué se debe la diferencia. Como Elster señala, «no sabemos cómo dar lugar a cambios de carácter duraderos y profundos. Además, el impacto de tales cambios sobre las propias experiencias emocionales dependerá fuertemente de acontecimientos externos y a veces impredecibles»33.

El logro de la autonomía y la libertad personal, incluyendo la libertad emocional, lo que alguien ha llamado la libertad del corazón 34 , es un proceso azaroso y de resultados inciertos. Sin embargo, sí que sabemos que determinadas circunstancias externas, como las libertades públicas y el respeto hacia distintas opciones vitales, aumentan la probabilidad del éxito en esa tarea, mientras que su ausencia opera en sentido contrario. Sobre ellas al menos podemos y hemos de actuar, si esa libertad es importante para nosotros.

33 Elster, Alchemies..., p. 414.

34 Cl. B. W. Helm, 'Freedom of the Heart', Pacific Philosophical Quarterly 77 (1996), pp. $71-87$. 